Check for updates

Cite this: RSC Adv., 2018, 8, 35840

\title{
Enhanced distance-dependent fluorescence quenching using size tuneable core shell silica nanoparticles $\uparrow$
}

\author{
Mohamed M. Elsutohy, (DD ab Amjad Selo, ${ }^{a}$ Veeren M. Chauhan, (DD ${ }^{a}$ Saul J. B. Tendler ${ }^{\text {ac }}$ \\ and Jonathan W. Aylott ${ }^{\star a}$
}

Silica nanoparticles (SNPs) have been used as favoured platforms for sensor, drug delivery and biological imaging applications, due to their ease of synthesis, size-control and bespoke physico-chemical properties. In this study, we have developed a protocol for the synthesis of size-tuneable SNPs, with diameters ranging from $20 \mathrm{~nm}$ to $500 \mathrm{~nm}$, through the optimisation of experimental components required for nanoparticle synthesis. This protocol was also used to prepare fluorescent SNPs, via covalent linkages of fluorophores, to the nanoparticle matrix using 3-aminopropyltriethoxysilane (APTES). This enabled the fabrication of ratiometric, fluorescent, $\mathrm{pH}$-sensitive nanosensors (75 $\mathrm{nm}$ diameter) composed SNPs covalently linked to two pH-sensitive fluorescent dyes Oregon Green (OG) and 5(6)carboxyfluorescein (FAM) and a reference fluorescent dye 5-(6)-carboxytetramethylrhodamine (TAMRA), extending the dynamic range of measurement from pH 3.5 to 7.5. In addition, size-tuneable, core-shell SNPs, covalently linked to a fluorescent TAMRA core were synthesised to investigate distance-dependant fluorescence quenching between TAMRA and black hole quencher 2 (BHQ2 ${ }^{\circledR}$ ) using nanometre-sized silica shells as physical spacers. The results showed a significant fluorescence quenching could be observed over greater distances than that reported for the classical distance-dependent molecular fluorescence quenching techniques, e.g. the Förster (fluorescence) resonance energy transfer (FRET). The methods and protocols we have detailed in this manuscript will provide the basis for the reproducible production of size tunable SNPs, which will find broad utility in the development of sensors for biological applications.

Received 11th July 2018

Accepted 6th October 2018

DOI: $10.1039 / c 8 r a 05929 b$

rsc.li/rsc-advances

\section{Introduction}

Nanoparticle-based techniques have opened new horizons towards the development of smart tools for many applications, including drug delivery, biological imaging and intracellular measurements. ${ }^{1}$

In particular, silica nanoparticles (SNPs) have been reported in a variety of studies due to their ease of synthesis, biocompatibility and versatile physicochemical properties. ${ }^{2,3}$ Synthesis of SNPs was firstly described by Stöber and co-workers ${ }^{4}$ in 1968 via hydrolysis and condensation of a silane alkoxide precursor, usually alcoholic tetraethylorthosilicate (TEOS), using ammonium hydroxide $\left(\mathrm{NH}_{4} \mathrm{OH}\right)$ as a catalyst, Fig. 1 .

${ }^{a}$ Boots Science Building, School of Pharmacy, University Park Campus, University of Nottingham, Nottingham, NG7 2RD, UK. E-mail: jon.aylott@nottingham.ac.uk ${ }^{b}$ Department of Pharmaceutical Analytical Chemistry, Faculty of Pharmacy, Al-Azhar University, Assiut 71524, Egypt

${ }^{c}$ Vice-Chancellor's Department, University of York, Heslington, York, YO10 5DD, UK † Electronic supplementary information (ESI) available. See DOI: 10.1039/c8ra05929b
During nanoparticle synthesis, a variety of components such as fluorescent molecules, drugs or sensing elements can be incorporated into the nanoparticle matrix to produce functionalised SNPs. ${ }^{5-8}$ For example, fluorescent SNPs that have been reported for biological imaging and sensing could be synthesised via covalent binding of fluorescent dye molecules to the nanoparticle matrix using a chemically active silane compound, 3-aminopropyltriethoxysilane (APTES) as a linker. ${ }^{9}$ In addition, the nanoparticle surface can be readily functionalised with several chemical groups to facilitate further surface conjugation, sensing and targeted therapy. ${ }^{10,11}$

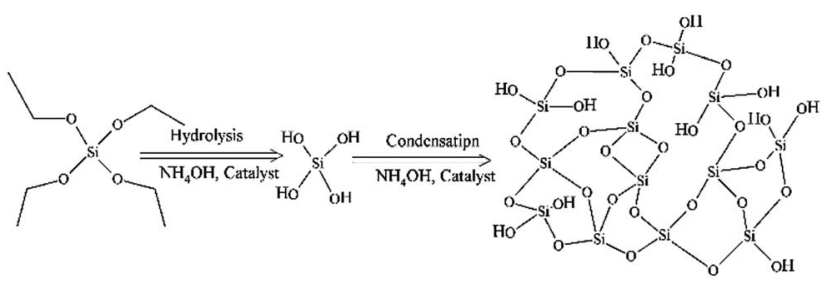

Fig. 1 A diagram illustrates the Stöber method for the synthesis of SNPs via hydrolysis and condensation of silica precursor (TEOS) using ammonium hydroxide $\left(\mathrm{NH}_{4} \mathrm{OH}\right)$ as a catalyst. 
Whilst many protocols have been reported for the synthesis of SNPs, there is a marked variation in the reported nanoparticle size from one study to another, based on the type and amount of reactant or catalyst used. ${ }^{\mathbf{1 2 - 1 5}}$ According to Stöber, different-sized SNPs can be fabricated through optimisation of the quantity of each reactant and catalyst required for nanoparticle synthesis. However, it is important to note previously reported studies ${ }^{\mathbf{1 2}, \mathbf{1 6}}$ do not agree with the findings reported by Stöber. These contradicting findings indicate the need for further studies to develop and optimise a valid protocol that can be readily followed for the synthesis of size-tuneable SNPs, especially for applications where nanoparticle size control is critical, e.g. intracellular measurements.

Furthermore, due to the ease of chemical functionalisation, silica has been used as an inert material to coat many systems, e.g. quantum dots and gold nanoparticles, producing core-shell structures surrounded by silica layers that can be readily functionalised. ${ }^{\mathbf{1 7 , 1 8}}$ These structures have been used to study the interaction between a fluorescent donor and an acceptor/ quencher, separated by nanometre-controlled distances, using the phenomena of the Förster (fluorescence) resonance energy transfer (FRET) and nanometal surface energy transfer (NSET), ${ }^{5,19}$ Fig. 2.

Changes in the recorded fluorescence signals of donoracceptor systems have been utilised as a sensing strategy in biosensor technology, viral diagnostics and for determination of distances between macromolecules (spectroscopic rulers). ${ }^{20-24}$ According to the literature, for an efficient FRET or NSET to occur the maximum distance between the fluorescent donor and its corresponding acceptor must be less than 10 or $20 \mathrm{~nm}$, respectively. ${ }^{25-27}$ However, recent studies have shown that an efficient fluorescence transfer/quenching was observed over longer distances than that reported. ${ }^{28-30}$ For example, Reineck et al. reported that NSET was observed between fluorophore molecules and gold nanoparticles over a distance of $38 \mathrm{~nm}$ using silica as physical spacers. ${ }^{31}$ This fluorescence transfer occurred when a silica shell was used as a separator between the donor and its acceptor; nevertheless, this fluorescence energy transfer/quenching was attributed to the plasmonic properties of gold nanoparticles due to the localised

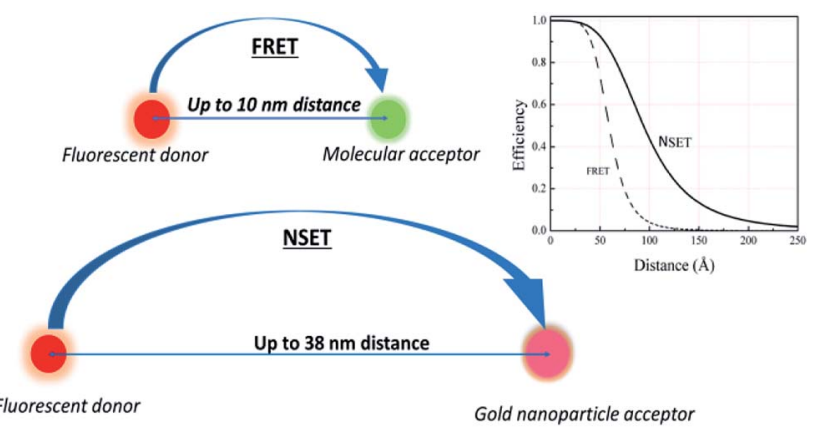

Fig. 2 Diagrammatic representation of the maximum distance reported for efficient Förster resonance energy transfer (FRET), occurring between molecular donor-acceptor system, and nanometal surface energy transfer (NSET), observed between a fluorescent donor and gold nanoparticle. surface plasmon resonance (LSPR), excluding any potential role that silica could exhibit. This reflects the fact that further research is required to investigate fluorescence transfer/ quenching between a fluorescent donor and a quencher over distances using silica shells as spacers.

In this study, we have developed a protocol optimising the amounts of each reactant and catalyst required for nanoparticle synthesis to produce size-controlled and tuneable SNPs. This protocol was applied to synthesise fluorescent SNPs covalently bounded to fluorophores using APTES. Ratiometric, fluorescent, pH-sensitive nanosensors and core-shell fluorescent SNPs were also fabricated. Fluorescence quenching between 5-(6)carboxytetramethylrhodamine (TAMRA) and black hole quencher 2 (BHQ2 $\left.{ }^{\circledR}\right)$, separated by silica shells with different thickness was observed and quantified.

\section{Results and discussion}

\section{Synthesis of size-controlled/tuneable SNPs}

Previously studies protocols have attempted to prepare SNPs; however, it was challenging to reproduce the reported particle diameters. ${ }^{\mathbf{1 3} \mathbf{1 4 , 3 2}}$ Therefore, the primary aim in our study was to develop a protocol to optimise the synthesis of size-tuneable SNPs that can be used in applications where nanoparticle size-control is important. The amounts of reactants and catalysts produces SNPs with variable sizes. ${ }^{33}$ Therefore, we started our study by investigating the influence of controlling the quantities of each reactant and catalyst (TEOS, $\mathrm{NH}_{4} \mathrm{OH}$ and ethanol) on the resulting nanoparticle diameter.

The interplay between varying volumes of TEOS (180-2000 $\mu \mathrm{L}, 98 \%$, equivalent to 0.80 to $8.8 \mathrm{mmol} \mathrm{L}^{-1}$ ) and nanoparticle diameter was investigated without changing the volume of $\mathrm{NH}_{4} \mathrm{OH}\left(1.0 \mathrm{~mL}\right.$ of $\left.28-30 \% \mathrm{v} / \mathrm{v}, 7.7 \mathrm{mmol} \mathrm{L}^{-1}\right)$ and ethanol (absolute 99\%, $16.75 \mathrm{~mL}$ ). The hydrodynamic diameter of each nanoparticle batch $(n=5)$ was measured using dynamic light scattering (DLS).

The results showed that different volumes of TEOS did not significantly $(p>0.05)$ influence the mean nanoparticle diameter, which were centred at $145 \pm 14 \mathrm{~nm}$ (polydispersity index, $(\mathrm{PDI}) \leq 1.04)$, Fig. 3. In addition, the recovered nanoparticles $(100 \pm 20 \mathrm{mg})$ were not influenced by increasing the volume of TEOS. As a result, $500 \mu \mathrm{L}\left(2.2 \mathrm{mmol} \mathrm{L}^{-1}\right)$ of TEOS was selected for all further experiments. In comparison, previously reported studies showed that increasing the concentration of TEOS resulted in an increase or occasionally a decrease in the nanoparticle diameter. ${ }^{12,16}$ The results obtained in this study are in agreement with that reported by Stöber, who concluded that varying amounts of TEOS did not influence the nanoparticle size. $^{4}$

In contrast, increasing the volumes of $\mathrm{NH}_{4} \mathrm{OH}(28-30 \% \mathrm{v} / \mathrm{v})$ from 0.3 to $5.0 \mathrm{~mL}\left(2.3\right.$ to $\left.38.5 \mathrm{mmol} \mathrm{L}^{-1}\right)$, whilst keeping the quantities of all other reactants constant (optimised TEOS of $500 \mu \mathrm{L}, 2.2 \mathrm{mmol} \mathrm{L}^{-1}$, and $16.75 \mathrm{~mL}$ of ethanol), gradually increased nanoparticle diameters from $20 \mathrm{~nm}$ to $200 \mathrm{~nm}$, Fig. 4A. Furthermore, by increasing the volume of $\mathrm{NH}_{4} \mathrm{OH}$ beyond $2.0 \mathrm{~mL}\left(15.4 \mathrm{mmol} \mathrm{L}^{-1}\right)$ did not significantly $(p>0.05)$ influence the nanoparticle diameter as it remained at $\sim 200 \mathrm{~nm}$. 


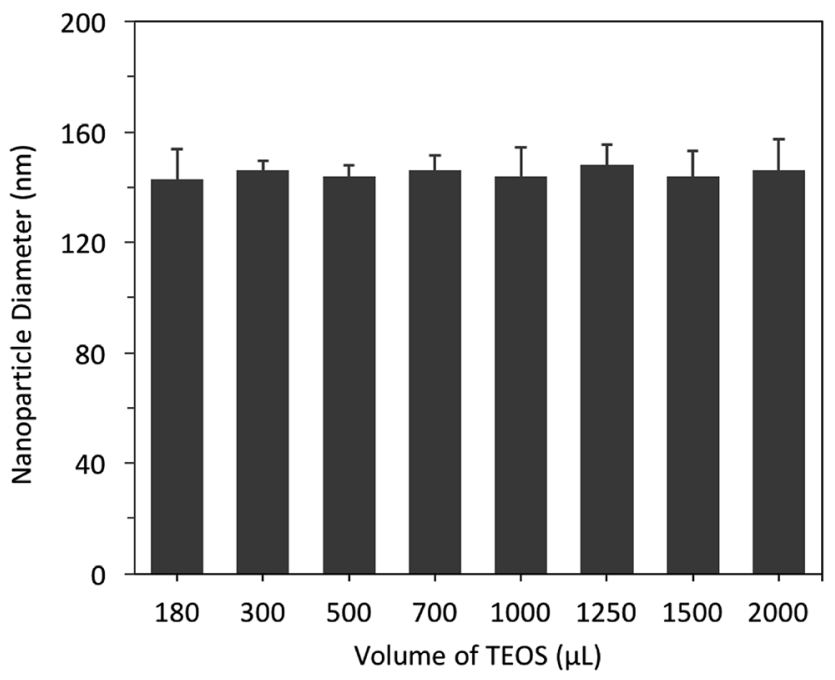

Fig. 3 The effect of controlling the volumes of TEOS $(180-2000 \mu \mathrm{L}$, $98 \%, 0.80$ to $8.8 \mathrm{mmol} \mathrm{L}^{-1}$ ) on the hydrodynamic diameter of SNPs, measured using DLS $(n=5)$.

With respect to nanoparticle yield, increasing the $\mathrm{NH}_{4} \mathrm{OH}$ volume increased the mass of nanoparticles recovered (see Table 1 in the ESI $\dagger$ ). Therefore, for the efficient separation of SNPs with a diameter $<50 \mathrm{~nm}$ membrane dialysis was used, whereas, freeze-drying with centrifugation was used to collect SNPs $>50 \mathrm{~nm}$.

DLS is an excellent methodology to determine nanoparticle sizes, nevertheless, it is important to combine more than one technique to further information on nanoparticle characteristics and confirm the results obtained from the DLS measurements. ${ }^{34}$ Therefore, transmission microscopy (TEM) was used to measure the nanoparticles size distribution and determine their morphology. Fig. 4B and C, shows the results obtained from TEM imaging and indicate that SNPs produced using different volumes of $\mathrm{NH}_{4} \mathrm{OH}$ were spherical in shape and had a narrow size distribution for all batches. The size measured using TEM was slightly smaller than that obtained from the DLS measurements. This is an expected observation as DLS measures the hydrodynamic diameter, which is the nanoparticle size combined with a surrounding solvent layer. ${ }^{36}$ Taken together, the results obtained from DLS agreed with TEM imaging.

To synthesise SNPs with a diameter greater than $200 \mathrm{~nm}$ we optimised a synthesis method that controls the volumes of $\mathrm{NH}_{4} \mathrm{OH}(0.3-5.0 \mathrm{~mL}$ of $28-30 \% \mathrm{v} / \mathrm{v})$ with constant volumes of TEOS $(\mu \mathrm{L})$ and ethanol $(16.75 \mathrm{~mL})$, in addition to the seed growth method that reported by Bogush et al. ${ }^{35}$ This procedure was based on a second addition of TEOS $\left(1.5 \mathrm{~mL}, 6.6 \mathrm{mmol} \mathrm{L}^{-1}\right)$ to the reaction mixture after the first 24 hours of the nanoparticle synthesis, prepared by using the different volumes of $\mathrm{NH}_{4} \mathrm{OH}$. The results showed that this two-step synthesis protocol produced monodisperse SNPs with diameters ranging from 200 to $500 \mathrm{~nm}$ (PDI < 0.10), Fig. 4D.

The effect of different volumes of alcohol solvent (ethanol) on nanoparticle diameter and size distribution were
A
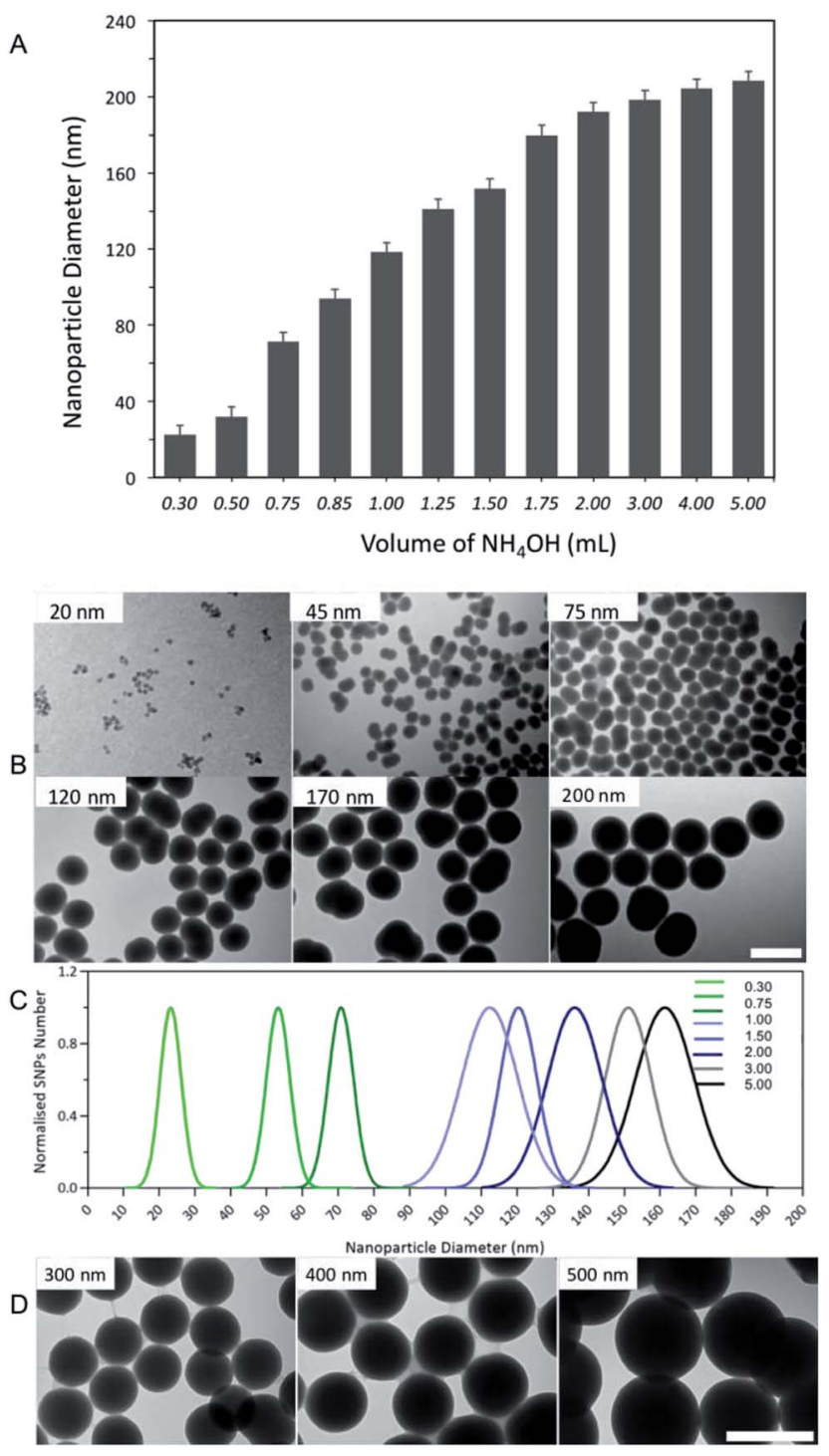

Fig. 4 (A) The interplay between different volumes of $\mathrm{NH}_{4} \mathrm{OH}(0.3$ to $5.0 \mathrm{~mL}(28-30 \%), 2.3$ to $38.5 \mathrm{mmol}^{-1}$ ) and SNPs diameter, measured by a DLS $(n=5)$. (B) TEM images of selected SNPs samples, produced using different volumes of $\mathrm{NH}_{4} \mathrm{OH}$, scale bar $=200 \mathrm{~nm}$. (C) TEM size distribution histogram of the resulting SNPs batches (D) TEM images of SNPs with diameters ranging from 200 to $500 \mathrm{~nm}$, synthesised using the optimised protocol combined with the seed growth method, ${ }^{35}$ scale bars $=500 \mathrm{~nm}$.

investigated. Firstly, SNPs were prepared by using variable volumes of ethanol, ranging from $10 \mathrm{~mL}$ to $50 \mathrm{~mL}$, and constant volumes of TEOS $(500 \mu \mathrm{L})$ and $\mathrm{NH}_{4} \mathrm{OH}(0.75 \mathrm{~mL})$. The results showed that increasing the volume of ethanol from 10 to $25 \mathrm{~mL}$ did not significantly $(p>0.05)$ influence the nanoparticle diameter, which remained at $70 \pm 18 \mathrm{~nm}$ (PDI $\leq 0.052$ ), Fig. 5 . By increasing the volume of ethanol beyond $25 \mathrm{~mL}$, but less than $50 \mathrm{~mL}$, subtly increased the nanoparticle diameter.

Secondly, the effect of alcohol type on nanoparticle diameter and size distribution was studied using either methanol or isopropanol $(16.75 \mathrm{~mL})$ instead of ethanol. The results revealed that using methanol for nanoparticle synthesis reduced nanoparticle size to $65 \mathrm{~nm}$. This diameter was smaller than that 


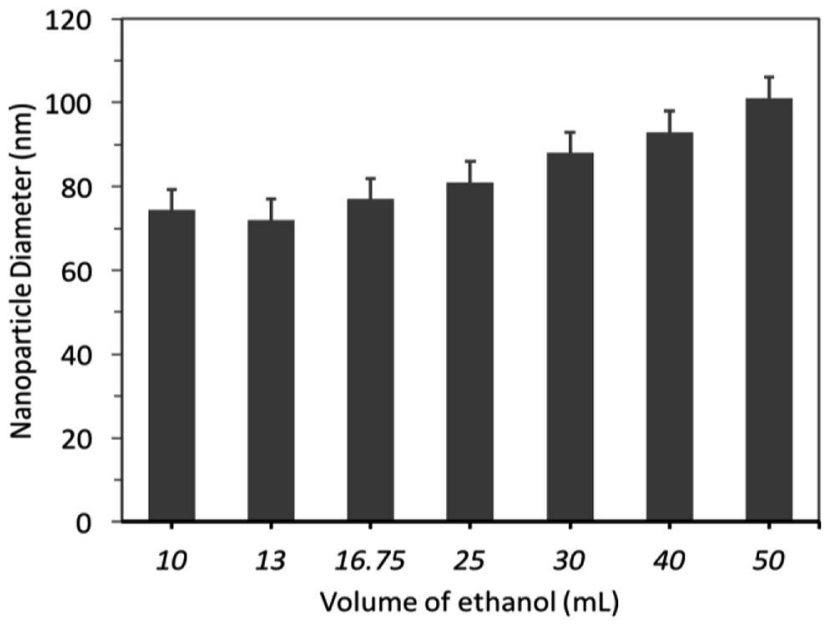

Fig. 5 The effect of different volumes of ethanol $(10-50 \mathrm{~mL})$ on SNPs diameter.

obtained using the same amount of ethanol $(75 \mathrm{~nm})$, however, methanol was excluded from further experiments due to potential toxicity and harmful environmental effects. ${ }^{37}$ Furthermore, polydisperse, large SNPs with a diameter of $220 \mathrm{~nm}$ and wide-size distribution were produced when isopropanol was used for nanoparticle synthesis. This could reflect the potential effect of alcohol polarity on nanoparticle size, aggregation and shape. ${ }^{38,39}$ Therefore, we used ethanol (16.75 $\mathrm{mL}$ ) as an alcoholic medium for nanoparticle synthesis.

Synthesis of SNPs can be explained by the von Weimarn and Ostwald ripening theories that describe how particles with a specific size are formed. ${ }^{40,41}$ According to these theories, for any particle to be formed, a supersaturated solution of the corresponding ions/molecules, hydrolysed TEOS in this case, is required to produce primary unstable small nuclei (nucleation). Once these primary nuclei reach equilibrium, they combine to form secondary, thermodynamically stable larger nuclei which spontaneously grow to form more stable and uniform particles. During SNPs synthesis, $\mathrm{NH}_{4} \mathrm{OH}$ is used as a catalyst to initiate the hydrolysis and condensation of TEOS, thus, using a constant volume of $\mathrm{NH}_{4} \mathrm{OH}$ with varying quantitates of TEOS does not influence the nanoparticle diameter as the number of the hydrolysed TEOS molecules is limited by the amount of $\mathrm{NH}_{4} \mathrm{OH}$. In contrast, increasing the quantity of $\mathrm{NH}_{4} \mathrm{OH}$ increases the rates of hydrolysis (nucleation) and condensation (particle growth), which in turn increases the number of hydrolysed TEOS molecules required for the primary nuclei formation and thus, increasing nanoparticle diameter, which could improve particle yield.

\section{Production of fluorescent SNPs and $\mathbf{p H}$-sensitive nanosensors}

Fluorescent SNPs $(75 \pm 8 \mathrm{~nm})$ were produced via covalent binding of fluorescent dye molecules to the nanoparticle matrix. Prior to nanoparticle synthesis, the succinimidyl ester derivative of fluorescent dye molecules were covalently linked to APTES (Fig. 6A) in an excess molar ratio of APTES : fluorescent dye $(1.2: 1)$ to ensure that all fluorophores were successfully conjugated to APTES. ${ }^{42,43}$ APTES-fluorescent dye conjugates

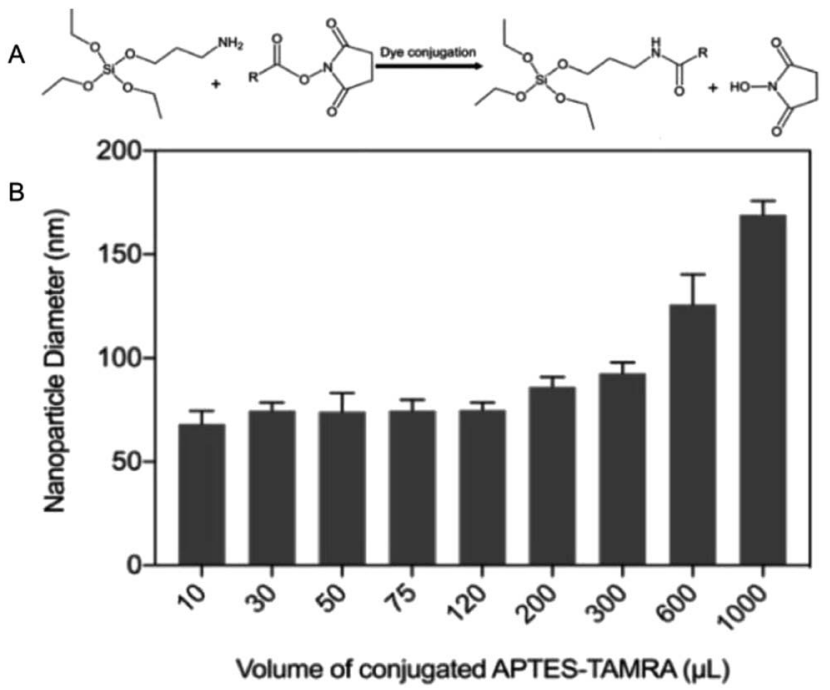

Fig. 6 (A) A schematic diagram shows the covalent conjugation reaction between the succinimidyl ester derivative of a fluorophore $(R)$ to APTES to prepare a conjugate that used for the synthesis of fluorescent SNPs. (B) The effect of APTES-TAMRA conjugate on nanoparticle diameter.

were added during nanoparticle synthesis to facilitate covalent linking of the fluorescent dye molecules to SNPs matrix and produce fluorescent SNPs. This experimental study was initiated by using 5-(and-6)-carboxytetramethylrhodamine (TAMRA) as an exemplar fluorophore to investigate the effect of varying volumes of APTES-fluorophore conjugate on nanoparticle diameter. The results showed that the hydrodynamic diameters of fluorescent SNPs were $75 \pm 13 \mathrm{~nm}$ for all batches, similar to that obtained with the non-fluorescent SNPs, provided that the volume of this conjugate were less than $300 \mu \mathrm{L}$. Further increase in TAMRA-APTES above volume greater than $300 \mu \mathrm{L}$, up to 1000 $\mu \mathrm{L}$, increased the nanoparticle diameter to $\sim 180 \mathrm{~nm}$, Fig. $6 \mathrm{~B}$.

In addition, the procedure, described for the synthesis of fluorescent SNPs, was also utilised to fabricate ratiometric, fluorescent, pH-sensitive, silica-based nanosensors. These nanosensors were composed SNPs ( $75 \mathrm{~nm}$ diameter) covalently linked to two pH-sensitive fluorescent dyes, Oregon Green (OG) and 5-(6)-carboxyfluorescein (FAM) in combination with TAMRA as a reference $\mathrm{pH}$-insensitive fluorophore to permit ratiometric measurements. Combination of such two $\mathrm{pH}$-sensitive fluorophores and optimising their ratio extended the effective dynamic range of nanosensors from $\mathrm{pH} 3.5$ to $7.5^{44}$ (see ESI $\dagger$ for further details).

\section{Fluorescence-quenching study using size-controlled fluorescent core-shell SNPs}

Silica has been previously reported as a spacer (shell) between a fluorescent molecule (donor) and noble-metal nanoparticles, such as gold, (acceptor or quencher) to investigate NSET or distance-dependent fluorescence transfer/quenching. ${ }^{26,27,31}$ To the best of our knowledge, molecular fluorescence quenching/ transfer between a fluorescent dye and black hole quenchers (BHQs) using silica spacers has not yet been studied. In this study, we conducted research to investigate fluorescence 
quenching between TAMRA (in the core) as a fluorescent emitter molecule and a corresponding molecular quencher (BHQ2) (linked to the surface) over different distances using silica shells as separators. BHQ2 was chosen as a selective molecular quencher for TAMRA, because it absorbs light at the same wavelength range of TAMRA fluorescence without reemission of the absorbed light. ${ }^{45}$ Three different models of TAMRA-SNPs and core-shell TAMRA-SNPs were synthesised and characterised, Fig. 7. Model 1 was composed of $75 \mathrm{~nm}$ TAMRA-labelled silica core. This fluorescent core was also used to synthesise model 2 and model 3 that were composed of a TAMRA labelled core (model 1) surrounded by nonfluorescent silica with thickness of $12.5 \mathrm{~nm}$ and $25 \mathrm{~nm}$, respectively (Fig. 7), as measured by TEM and confirmed by DLS measurements. Binding of BHQ2 to the nanoparticle model surface was performed using the EDC/NHS chemistry of molecular conjugation (see ESI $\dagger$ ).

To study the fluorescence quenching over the specified separation distances of the developed silica models, the fluorescence signal of each model was measured in comparison to fluorescent TAMRA-SNPs that were not linked to BHQ2. The percentage of fluorescence quenching was calculated using eqn (1):

$$
Q(\%)=\frac{F-F_{\mathrm{q}}}{F} \times 100
$$

where $Q$ is the percentage of fluorescence quenching, $F$ is the fluorescence intensity of TAMRA-SNPs (without BHQ2), and $F_{\mathrm{q}}$ is the fluorescence intensity after modification and binding to BHQ2.

The results showed that fluorescence quenching of TAMRA was observed in all models after binding to BHQ2, compared to the corresponding blank sample of each model, Fig. 8. While the relationship between $\mathrm{BHQ} 2$ concentration and fluorescence quenching of TAMRA was established using model 1 using different concentrations of BHQ2, linked to nanoparticle surface. This relationship was similar to a typical Beer-Lambert calibration curve, which indicates that fluorescence quenching is dependent on $\mathrm{BHQ} 2$ concentration while further increase in

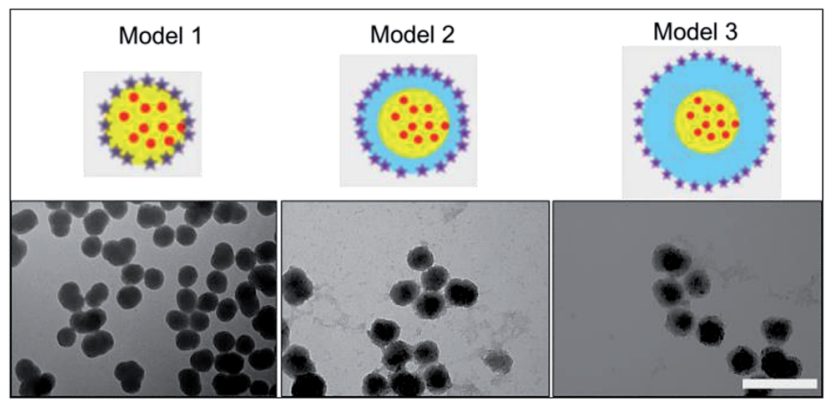

Fig. 7 Diagrammatic representation of model 1, model 2 and 3 and their corresponding TEM images. Red dots within the yellow core illustrates the 75 TAMRA-SNPs while the stars on the surface express BHQ2 molecules, linked to nanoparticle surface, the blue layer illustrates the plain non-fluorescent silica shell surrounding the fluorescent core with thickness of $\sim 12.5 \mathrm{~nm}$ and $25 \mathrm{~nm}$ for model 2 \& 3 respectively. Scale bar $=200 \mathrm{~nm}$.
BHQ2 quantity above $0.20 \mu \mathrm{mol} \mathrm{L}^{-1}$ did not influence the percentage of TAMRA quenching (Fig. 8A). As a result, $0.25 \mu \mathrm{mol}$ $\mathrm{L}^{-1}$ of $\mathrm{BHQ} 2$ was selected as the optimised concentration that provided maximum fluorescence quenching of TAMRA (Fig. 8B). The maximum fluorescence quenching of model 1, model 2 or model 3 was $\sim 75 \%, 45 \%$ or $25 \%$ respectively, (Fig. 8C).

Further investigations were performed to study the possibility of whether BHQ2 diffusion through the silica matrix could interfere with the obtained fluorescence quenching. To conduct this experiment, model 1 was prepared without any surface functionalisation or activation, required to bind $\mathrm{BHQ} 2$, and stirred overnight with BHQ2 $\left(0.25 \mu \mathrm{mol} \mathrm{L}^{-1}\right)$. After nanoparticle separation, the fluorescence signal of TAMRA was detected and compared to that recorded for the corresponding nanoparticle sample, treated similarly without mixing with BHQ2. The results revealed that the fluorescence intensity of TAMRA of the non-functionalised model 1 and blank sample were not statistically different ( $p>0.05)$ (Fig. S4, ESI $\dagger$ ). This indicates minimal diffusion of BHQ2 through the silica matrix and confirms that observed fluorescence quenching of the developed models is due to BHQ2 that is chemically linked to the nanoparticle surface and such variable quenching can be due to changes in the separation distance.

In our systems, the fluorescence quenching observed in model 1 (75\%, no spacer layer) fits with FRET theory where the distance between donor and acceptor is $<10 \mathrm{~nm}$. We would anticipate some fluorescence being emitted from the model 1 core as the average particle size is $75 \mathrm{~nm}$ and so not all fluorophores will be within the FRET distance limits. In addition to fluorescence quenching there may be a contribution to the reduced fluorescence output via re-absorption of TAMRA emission by BHQ2. Our findings for models $2 \& 3$ highlight that fluorescence quenching occurs beyond the $10 \mathrm{~nm}$ distance limit accepted for efficient fluorescence resonance energy transfer (FRET) between donor and quencher molecules ${ }^{5,18,45}$ (Fig. 2). For our engineered SNPs (models 2 \& 3) where TAMRA and BHQ2 were separated by silica shells with thickness of approximately 12.5 and $25 \mathrm{~nm}$, respectively, conventional FRET theory does not explain the quenching observed as the separation distance is greater than $10 \mathrm{~nm}$. While energy transfer between a fluorescent donor and a gold nanoparticle quencher across a silica spacer layer has previously been observed over an extended distance, this has been attributed to NSET. ${ }^{30}$ Taken together, we postulate that the observed quenching is due to a combination of energy transfer, emission re-absorption and a possible influence of the silica shell (similar to that hypothesised by Reineck et al. ${ }^{31}$ ).

While the mechanism behind this observed distancedependent fluorescence quenching has not yet been confirmed, fluorescence quenching over this extended distance could provide a new sensing strategy where varying the distance between the fluorescence donor-acceptor system is used for detection. This could include the monitoring of DNA hybridisation and the development of aptamer-based biosensors (aptasensors) for the detection of biomarkers, e.g. ATP, thrombin and adenosine. ${ }^{\mathbf{4 6}}$ 

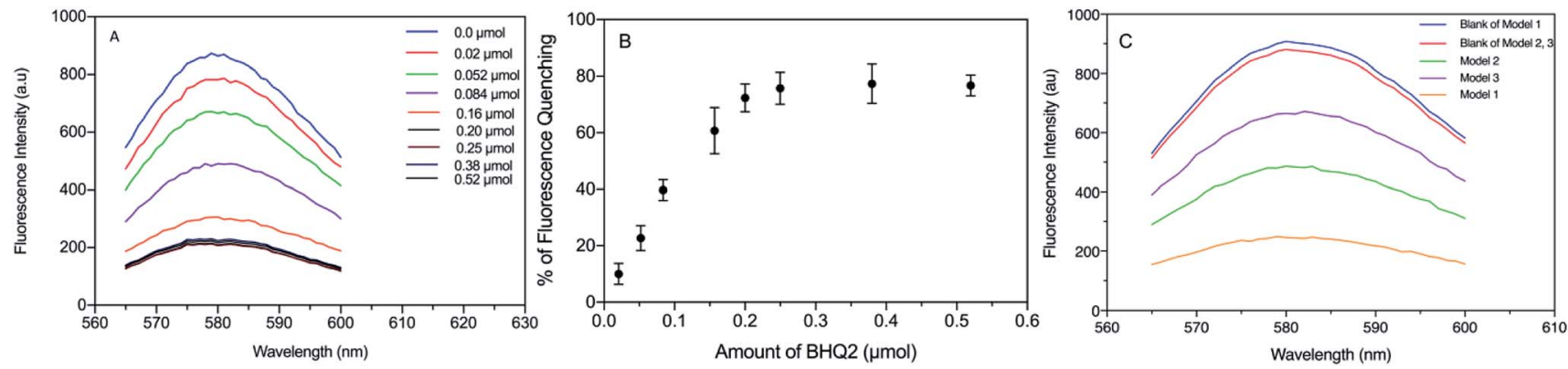

Fig. 8 (A) Fluorescence spectra of model 1, linked to different concentrations of BHQ2 and compared to a blank (without BHQ2 molecules). (B) Calibration curve for the percentage of fluorescence quenching resulted from model $1(n=5)$ and a range of BHQ2 concentrations. (C) Fluorescence spectra of model 1, 2 and 3 after binding to BHQ2 $\left(0.25 \mu \mathrm{mol} \mathrm{L}{ }^{-1}\right)$, compared to a corresponding blank (SNPs model prepared without BHQ2)

\section{Conclusion}

In this study, a simple protocol for the synthesis of size-tuneable SNPs, with diameters ranging from 20 to $500 \mathrm{~nm}$, has been developed by optimising the amount of each reactant and catalyst that are required for nanoparticle synthesis. The results showed that $\mathrm{NH}_{4} \mathrm{OH}$ plays a key role in controlling the size of SNPs and by careful selection of the volume of $\mathrm{NH}_{4} \mathrm{OH}$ the nanoparticle diameter can be varied from $20 \mathrm{~nm}$ to $200 \mathrm{~nm}$. Through selection of optimised $\mathrm{NH}_{4} \mathrm{OH}$ volumes and subsequent addition of TEOS, the nanoparticle diameters were controllably increased from $200 \mathrm{~nm}$ to $500 \mathrm{~nm}$. In addition, SNPs covalently linked to fluorescent dye molecules were produced. This enabled the fabrication of ratiometric, fluorescent $\mathrm{pH}$-sensitive nanosensors. Furthermore, using the versatile chemistry and sizetunability of SNPs, three models of fluorescent-quencher SNPs were synthesised and used to investigate fluorescence quenching between fluorophore molecules (TAMRA) and a molecular black hole quencher (BHQ2). The results showed a significant distancedependent fluorescence quenching over a greater distance than that expected, which could be used as an analytical technique in sensing applications, e.g. nanosensors where the fluorescence signal is distant dependent.

\section{Experimental}

\section{Materials and chemicals}

The succinimidyl ester derivatives of Oregon Green ${ }^{\circledR} 488$ carboxylic acid 5-isomer (OG), 5-(6)-carboxyfluorescein (FAM) and 5-(and-6)-carboxytetramethylrhodamine (TAMRA) were obtained from Invitrogen, USA. Tetraethylorthosilicate (TEOS, 99\%), 3-aminopropyltriethoxysilane 98\% (APTES), citric acid monohydrate, sodium dibasic phosphate, phosphate buffer saline tablets (PBS, pH 7.4), 1-ethyl-3-(3-dimethylaminopropyl)$\mathrm{N}$-carbodiimide hydrochloride (EDC) and $N$-hydroxysuccinimide (NHS) were purchased from Sigma-Aldrich, UK. Ammonium hydroxide solution $\left(\mathrm{NH}_{4} \mathrm{OH}, 28-30 \%\right)$ was obtained from Acros Organics, USA. Ethanol absolute (99.5\%) and 2-( $n$ morpholino)ethanesulfonic acid (MES, 0.2 M, pH 5.7) were obtained from Fisher Scientific, UK. Amine-modified black hole quencher $2{ }^{\circledR} \quad$ (BHQ2) was purchased from Biosearch
Technologies, USA. Carboxyethylsilanetriol sodium salt, $25 \% \mathrm{~g}$ $\mathrm{g}^{-1}$ was purchased from abcrGmbH, Germany.

\section{Synthesis of size-tuneable silica nanoparticles}

Into a scintillation vial, $16.75 \mathrm{~mL}$ of absolute ethanol was mixed $\mathrm{NH}_{4} \mathrm{OH}(28-30 \% \mathrm{w} / \mathrm{v})$ with a volume specified in Table $1(\mathrm{ESI} \dagger)$ to prepare SNPs with diameters ranging from 20 to $200 \mathrm{~nm}$. Following this, $500 \mu \mathrm{L}$ of TEOS was added dropwise to this mixture (ethanol- $\mathrm{NH}_{4} \mathrm{OH}$ ), with continuous stirring for 24 hours at room temperature. To collect SNPs, dialysis and freeze drying was used for nanoparticles $<50 \mathrm{~nm}$ while centrifugation was used to collect SNPs $>50 \mathrm{~nm}$ at $6000 \mathrm{rpm}$ for 30 minutes, followed by washing for three times with $30 \mathrm{~mL}$ of absolute ethanol. After the final wash, the suspensions containing SNPs were transferred into a $50 \mathrm{~mL}$ rounded bottom flask to evaporate ethanol using a rotary evaporator at $30{ }^{\circ} \mathrm{C}$ and the dried nanoparticles were collected and stored at $4{ }^{\circ} \mathrm{C}$. To prepare SNPs with nanoparticle diameter larger than $200 \mathrm{~nm}$, the same procedure described above was used followed by further dropwise addition of $1.5 \mathrm{~mL}$ of TEOS after the first 24 hours to each vial containing the specified amounts of $\mathrm{NH}_{4} \mathrm{OH}$, with continuous stirring for further 24 hours at room temperature. Following this, nanoparticle collection, purification and drying were performed as described using centrifugation.

\section{Synthesis of fluorescent SNPs, pH nanosensors, core-shell models}

Prior to nanoparticle synthesis, fluorophore conjugation to APTES was performed ${ }^{43}$ using an accurately weighed $1 \mathrm{mg}$ of the succinimidyl ester derivative of each fluorophore (OG, FAM or TAMRA), dissolved separately into $1 \mathrm{~mL}$ of absolute ethanol, stirred overnight with $20 \mu \mathrm{L}$ of APTES (98\%), in the dark and stored in the dark at $4{ }^{\circ} \mathrm{C}$ until further. The protocol for the synthesis of SNPs was followed using $0.75 \mathrm{~mL}$ of $\mathrm{NH}_{4} \mathrm{OH}$, before the addition of TEOS $(500 \mu \mathrm{L})$, a volume of fluorophore-APTES conjugates $(100 \mu \mathrm{L})$, was added dropwise to the ethanol- $\mathrm{NH}_{4} \mathrm{OH}$ mixture with continuous stirring for 24 hours in the dark. Following this, $500 \mu \mathrm{L}$ of TEOS was added dropwise to the reaction mixture with stirring for further 24 hours and the same procedure for SNPs synthesis was followed. 
To fabricate the pH-sensitive nanosensors. $40 \mu \mathrm{L}$ of $\mathrm{OG}-$ APTES, $40 \mu \mathrm{L}$ of FAM-APTES and $60 \mu \mathrm{L}$ of TAMRA-APTES were used and the procedure for the synthesis of fluorescent SNPs, described above, was followed.

Synthesis of model 1, 2 and 3. Model 1 was synthesised using APTES-TAMRA conjugate $(20 \mu \mathrm{L})$, added dropwise to the ethanol- $\mathrm{NH}_{4} \mathrm{OH}$ mixture, prepared using $5.8 \mathrm{mmol} \mathrm{L}^{-1}, 0.75$ $\mathrm{mL}$. The same procedure for the synthesis of fluorescent SNPs was followed. For the preparation of model 2 and 3, the same nanoparticle batch of model 1 was used using an accurately $10 \mathrm{mg}$ of model 1 , transferred into separate scintillation vials and suspended into $1 \mathrm{~mL}$ of absolute ethanol. Two plain silica shells, with different thickness, were added to model 1 using either $30 \mu \mathrm{L}$ or $50 \mu \mathrm{L}$ of $\mathrm{NH}_{4} \mathrm{OH}(28-30 \% \mathrm{w} / \mathrm{v})$, added to the scintillation vials containing the $10 \mathrm{mg}$ of model 1 , with continuous oscillation, followed by consecutive three additions of $17 \mu \mathrm{L}$ of TEOS with 15 minutes time intervals between each addition, as reported. ${ }^{47}$ After 24 hours, the contents of the vials were centrifuged and washed three times with $1 \mathrm{~mL}$ of PBS, $\mathrm{pH}$ 7.4 and nanoparticles were collected and used for surface modification, activation and binding to $\mathrm{BHQ} 2$, as described below.

\section{Carboxylic acid surface modification, EDC/NHS activation and binding to amine-BHQ2 ${ }^{47}$}

To modify the nanoparticle surface of model 1 , accurately weighed $10 \mathrm{mg}$ of the $75 \mathrm{~nm}$ TAMRA-SNPs was suspended into $1 \mathrm{~mL}$ of PBS buffer. Model 2 and 3, were directly used after their preparation as in the previous step. Following this, $40 \mu \mathrm{L}$ of carboxyethylsilanetriol sodium salt $\left(25 \% \mathrm{~g} \mathrm{~g}^{-1}\right)$ was added to each vial containing the corresponding nanoparticle model and the mixtures were gently stirred for 24 hours in the dark. The resultant carboxylic acid modified SNPs were centrifuged and washed with $1 \mathrm{~mL}$ of PBS for three times while the last wash was performed using $1 \mathrm{~mL}$ of $0.2 \mathrm{M}$ MES buffer, $\mathrm{pH}$ of 5.7. Following this, $30 \mu \mathrm{L}$ of freshly prepared EDC and NHS solutions $(500 \mathrm{mg}$ $\mathrm{mL}^{-1}$ prepared separately in MES buffer, $\mathrm{pH}$ 5.7) were added to each vial (model). The reaction mixtures were stirred for 30 minutes followed by centrifugation and washing, three times, with $1 \mathrm{~mL}$ PBS buffer.

For BHQ2 binding, $120 \mu \mathrm{L}$ of BHQ2 $\left(1 \mathrm{mg} \mathrm{mL}{ }^{-1}\right.$, prepared in ethanol) was added to the EDC/NS activated SNPs models, with continuous stirring for 24 hours, followed by centrifugation and several washings with $1 \mathrm{~mL}$ of PBS. The nanoparticle suspension of each model, linked to BHQ2, were collected in PBS (1 $\mathrm{mL}$ ) and used for measurement of fluorescence intensity/ quenching of TAMRA, against blank samples of each batch treated similarly without binding to BHQ2.

\section{Nanoparticle/nanosensor characterisation}

The hydrodynamic diameter and zeta potential measurements were performed using Malvern Zetasizer Nano II DLS instrument. For the hydrodynamic diameter measurements, A $20 \mu \mathrm{L}$ of the original nanoparticle suspensions was diluted with $3 \mathrm{~mL}$ of deionised water. Zeta potential measurements were performed using a zeta potential cuvette of $0.1 \mathrm{mg} \mathrm{mL}^{-1}$ of SNPs suspended into $1 \mathrm{~mL}$ of $10 \% \mathrm{v} / \mathrm{v}$ PBS. Transmission electron microscopy (TEM) (FEI Tecnai 12, Biotwin) imaging was performed using $0.1 \mathrm{mg} \mathrm{mL} \mathrm{m}^{-1}$ of nanoparticle suspension, prepared in water, at a voltage of $100 \mathrm{kv}$. All the TEM images were analysed using ImageJ software to determine the median diameter and size distribution. All fluorescence measurements were performed using a Varian Cary Eclipse fluorescence spectrophotometer at the selected excitation and emission maxima of each fluorophore. For nanosensors calibration, $0.5 \mathrm{mg} \mathrm{mL}^{-1}$ of nanosensors were suspended in citric acid monohydrate/ sodium dibasic phosphate buffer, prepared with different $\mathrm{pH}$ values from $\mathrm{pH}$ 2.5-8.5 and fluorescence measurements were performed. Fluorescence intensities of OG and FAM were detected at $520 \mathrm{~nm}$ after excitation at $488 \mathrm{~nm}$ while the fluorescence intensity or quenching of TAMRA was recorded at $577 \mathrm{~nm}$ after excitation at $545 \mathrm{~nm}$. The ratio between the fluorescence intensity of OG/FAM and TAMRA, detected in the different $\mathrm{pH}$ values, was calculated and used to construct the ratiometric fluorescence calibration curve, against blank buffer samples.

To investigate the fluorescence quenching using SNPs models, the relationship between varying amounts of BHQ2 and the fluorescence intensity of model 1 was recorded after binding of the EDC/NHS activated SNPs to different amounts of BHQ2 ( 0.02 to $0.52 \mu \mathrm{mol} \mathrm{L}{ }^{-1}, 10$ to $250 \mu \mathrm{L}$ of $1 \mathrm{mg} \mathrm{mL}^{-1}$ ), followed by centrifugation and washing as described previously. The fluorescence quenching of TAMRA-SNPs after binding to BHQ2 was measured against blank samples treated similarly without the reaction with $\mathrm{BHQ} 2$. All fluorescence quenching measurements were detected for the corresponding SNPs/core-shell model against blank sample treated similarly and the percentage of fluorescence quenching was calculated.

\section{Author contributions}

ME developed the protocol for the preparation of size tuneable nanoparticles, synthesised the $\mathrm{pH}$-sensitive nanosensors and conducted DLS and fluorescence measurements. AS produced size-tuneable silica nanoparticles using the protocol developed and conducted TEM analysis (size tuneable and core shell particles). ME, AS and VMC prepared figures and tables. ME produced core-shell particles and optimised quenching experiments. ME, VMC, SJBT and JWA prepared manuscript. ME and VMC produced ESI. $\dagger$ ME, VMC, SJBT and JWA edited manuscript for publication. All authors contributed to scientific planning and direction.

\section{Conflicts of interest}

The authors declare no conflict of interest.

\section{Acknowledgements}

This work was supported by the Engineering and Physical Sciences Research Council [grant number EP/L01646X, EP/ P006485/1], the first EPSRC grant to AS through the Centre of Doctoral Training-Advance Therapeutics and Nanomedicine; 
the second EPSRC grant to VMC and JWA for the Future Targeted Healthcare Manufacturing Hub. Financial and in-kind support from the consortium of industrial users is also acknowledged (VMC, JWA). This research was funded by the Islamic Development Bank (ME). The authors also thank the Nanoscale and Microscale Research Centre (nmRC) for providing access to instrumentation.

\section{References}

1 T. Vo-Dinh, Nanotechnology in Biology and Medicine: Methods, Devices and Applications, Taylor and Francis Group, CRC Press, New york, USA, 2007, vol. 1, pp. 1-8.

2 Z. Li, J. C. Barnes, A. Bosoy, J. F. Stoddart and J. I. Zink, Mesoporous silica nanoparticles in biomedical applications, Chem. Soc. Rev., 2012, 41(7), 2590-2605.

3 A. Burns, H. Ow and U. Wiesner, Fluorescent core-shell silica nanoparticles: towards "Lab on a Particle" architectures for nanobiotechnology, Chem. Soc. Rev., 2006, 35(11), 10281042.

$4 \mathrm{~W}$. Stober, A. Fink and E. Bohn, Controlled Growth of Monodisperse Silica Spheres in the Micron Size Range, $J$. Colloid Interface Sci., 1968, 26, 62-69.

5 L. Wang and W. H. Tan, Multicolor FRET silica nanoparticles by single wavelength excitation, Nano Lett., 2006, 6(1), 84-88.

6 I. I. Slowing, J. L. Vivero-Escoto, C. W. Wu and V. S. Y. Lin, Mesoporous silica nanoparticles as controlled release drug delivery and gene transfection carriers, Adv. Drug Delivery Rev., 2008, 60(11), 1278-1288.

7 B. Y. Liu, F. Zeng, S. Z. Wu, J. S. Wang and F. C. Tang, Ratiometric sensing of mercury(II) based on a FRET process on silica core-shell nanoparticles acting as vehicles, Microchim. Acta, 2013, 180(9-10), 845-853.

8 T. Doussineau, S. Trupp and G. J. Mohr, Ratiometric pHnanosensors based on rhodamine-doped silica nanoparticles functionalized with a naphthalimide derivative, J. Colloid Interface Sci., 2009, 339(1), 266-270.

9 V. M. Chauhan, R. H. Hopper, S. Z. Ali, E. M. King, F. Udrea, C. H. Oxley and J. W. Aylott, Thermo-optical characterization of fluorescent rhodamine $\mathrm{B}$ based temperature-sensitive nanosensors using a CMOS MEMS micro-hotplate, Sens. Actuators, B, 2014, 192, 126-133.

10 Y. Zhang, B. Y. Hsu, C. Ren, X. Li and J. Wang, Silica-based nanocapsules: synthesis, structure control and biomedical applications, Chem. Soc. Rev., 2015, 44(1), 315-335.

11 B. I. Tamba, A. Dondas, M. Leon, A. N. Neagu, G. Dodi, C. Stefanescu and A. Tijani, Silica nanoparticles: preparation, characterization and in vitro/in vivo biodistribution studies, Eur. J. Pharm. Sci., 2015, 71, 46-55.

12 K. S. Rao, K. El-Hami, T. Kodaki, K. Matsushige and K. Makino, A novel method for synthesis of silica nanoparticles, J. Colloid Interface Sci., 2005, 289(1), 125-131.

13 G. Canton, R. Riccò, F. Marinello, S. Carmignato and F. Enrichi, Modified Stöber synthesis of highly luminscent dye-doped silica nanoparticles, J. Nanopart. Res., 2011, 13, 4349-4356.
14 M.-C. Estévez, M. B. O'Donoghue, X. Chen and W. Tan, Highly Fluorescent Dye-Doped Silica Nanoparticles Increase Flow Cytometry Sensitivity for Cancer Cell Monitoring, Nano Res., 2009, 2, 448-461.

15 H. Ow, D. R. Larson, M. Srivastava, B. A. Baird, W. W. Webb and U. Wiesner, Bright and stable core-shell fluorescent silica nanoparticles, Nano Lett., 2005, 5(1), 113-117.

16 J. W. Kim, L. U. Kim and C. K. Kim, Size Control of Silica Nanoparticles and Their Surface Treatment for Fabrication of Dental Nanocomposites, Biomacromolecules, 2007, 8, 215-222.

17 B. T. Ji, E. Giovanelli, B. Habert, P. Spinicelli, M. Nasilowski, X. Z. Xu, N. Lequeux, J. P. Hugonin, F. Marquier, J. J. Greffet and B. Dubertret, Non-blinking quantum dot with a plasmonic nanoshell resonator, Nat. Nanotechnol., 2015, 10(2), 170-175.

18 H. L. Ding, Y. X. Zhang, S. Wang, J. M. Xu, S. C. Xu and G. H. Li, Fe3O4@SiO2 Core/Shell Nanoparticles: The Silica Coating Regulations with a Single Core for Different Core Sizes and Shell Thicknesses, Chem. Mater., 2012, 24(23), 4572-4580.

19 L. Bertry, D. Olivier, P. Aschehoug, B. Viana and C. Chanéac, Experimental evidence of luminescence quenching at long coupling distances in europium (III) doped core-shell gold silica nanoparticles, Gold Bull., 2013, 46(4), 349-355.

20 F. Gao, P. Cui, X. X. Chen, Q. Q. Ye, M. G. Li and L. Wang, A DNA hybridization detection based on fluorescence resonance energy transfer between dye-doped core-shell silica nanoparticles and gold nanoparticles, Analyst, 2011, 136(19), 3973-3980.

21 W. B. Qiang, H. P. Liu, W. Li, X. Chen and D. K. Xu, Labelfree detection of adenosine based on fluorescence resonance energy transfer between fluorescent silica nanoparticles and unmodified gold nanoparticles, Anal. Chim. Acta, 2014, 828, 92-98.

22 Y. Chen, M. B. O'Donoghue, Y. F. Huang, H. Z. Kang, J. A. Phillips, X. L. Chen, M. C. Estevez, C. Y. J. Yang and W. H. Tan, A Surface Energy Transfer Nanoruler for Measuring Binding Site Distances on Live Cell Surfaces, $J$. Am. Chem. Soc., 2010, 132(46), 16559-16570.

23 T. Sen, S. Sadhu and A. Patra, Surface energy transfer from rhodamine 6G to gold nanoparticles: A spectroscopic ruler, Appl. Phys. Lett., 2007, 91(4), 043104.

24 J. Griffin, A. K. Singh, D. Senapati, P. Rhodes, K. Mitchell, B. Robinson, E. Yu and P. C. Ray, Size- and DistanceDependent Nanoparticle Surface-Energy Transfer (NSET) Method for Selective Sensing of Hepatitis C Virus RNA, Chem.-Eur. J., 2009, 15(2), 342-351.

25 C. Berney and G. Danuser, FRET or no FRET: A quantitative comparison, Biophys. J., 2003, 84(6), 3992-4010.

26 C. J. Breshike, R. A. Riskowski and G. F. Strouse, Leaving Forster Resonance Energy Transfer Behind: Nanometal Surface Energy Transfer Predicts the Size-Enhanced Energy Coupling between a Metal Nanoparticle and an Emitting Dipole, J. Phys. Chem. C, 2013, 117(45), 23942-23949.

27 C. S. Yun, A. Javier, T. Jennings, M. Fisher, S. Hira, S. Peterson, B. Hopkins, N. O. Reich and G. F. Strouse, 
Nanometal surface energy transfer in optical rulers, breaking the FRET barrier, J. Am. Chem. Soc., 2005, 127(9), 3115-3119.

28 W. R. Algar, K. Susumu, J. B. Delehanty and I. L. Medintz, Semiconductor Quantum Dots in Bioanalysis: Crossing the Valley of Death, Anal. Chem., 2011, 83(23), 8826-8837.

29 A. Samanta, Y. Zhou, S. Zou, H. Yan and Y. Liu, Fluorescence quenching of quantum dots by gold nanoparticles: a potential long range spectroscopic ruler, Nano Lett., 2014, 14(9), 5052-5057.

30 F. Gao, Q. Ye, P. Cui and L. Zhang, Efficient fluorescence energy transfer system between CdTe-doped silica nanoparticles and gold nanoparticles for turn-on fluorescence detection of melamine, J. Agric. Food Chem., 2012, 60(18), 4550-4558.

31 P. Reineck, D. Gomez, S. H. Ng, M. Karg, T. Bell, P. Mulvaney and U. Bach, Distance and Wavelength Dependent Quenching of Molecular Fluorescence by Au@SiO2 CoreShell Nanoparticles, ACS Nano, 2013, 7(8), 6636-6648.

32 L. M. Rossi, L. Shi, F. H. Quina and Z. Rosenzweig, Stober synthesis of monodispersed luminescent silica nanoparticles for bioanalytical assays, Langmuir, 2005, 21(10), 4277-4280.

33 D. Guarnieri, M. A. Malvindi, V. Belli, P. P. Pompa and P. Netti, J. Nanopart. Res., 2014, 16, 1-14.

34 H. Fissan, S. Ristig, H. Kaminski, C. Asbach and M. Epple, Comparison of different characterization methods for nanoparticle dispersions before and after aerosolization, Anal. Methods, 2014, 6(18), 7324.

35 G. H. Bogush, M. A. Tracy and C. F. Zukoski, Preparation of Monodisperse Silica Particles - Control of Size and Mass Fraction, J. Non-Cryst. Solids, 1988, 104(1), 95-106.

36 A. S. Desai, V. M. Chauhan, A. P. Johnston, T. Esler and J. W. Aylott, Fluorescent nanosensors for intracellular measurements: synthesis, characterization, calibration, and measurement, Front. Physiol., 2013, 4, 401.
37 T. R. Tephly, The toxicity of methanol, Life Sci., 1991, 48(11), 1031-1041.

38 I. Sang Hyuk, T. Herricks, Y. Tack Lee and Y. Xia, Synthesis and characterization of monodisperse silica colloids loaded with superparamagnetic iron oxide nanoparticles, Chem. Phys. Lett., 2005, 401(1-3), 19-23.

39 Y.-H. Deng, C.-C. Wang, J.-H. Hu, W.-L. Yang and S.-K. Fu, Investigation of formation of silica-coated magnetite nanoparticles via sol-gel approach, Colloids Surf., A, 2005, 262(1-3), 87-93.

40 P. P. v. Weimarn, The Precipitation Laws, Chem. Rev., 1925, 2(2), 217-242.

41 W. Ostwald, Z. Phys. Chem., 1897, 22, 289-330.

42 O. S. Wolfbeis, An overview of nanoparticles commonly used in fluorescent bioimaging, Chem. Soc. Rev., 2015, 44(14), 4743-4768.

43 X. Chen, M. C. Estevez, Z. Zhu, Y. F. Huang, Y. Chen, L. Wang and W. Tan, Using aptamer-conjugated fluorescence resonance energy transfer nanoparticles for multiplexed cancer cell monitoring, Anal. Chem., 2009, 81(16), 70097014.

44 M. M. Elsutohy, V. M. Chauhan, R. Markus, M. A. Kyyaly, S. J. B. Tendler and J. W. Aylott, Real-time measurement of the intracellular $\mathrm{pH}$ of yeast cells during glucose metabolism using ratiometric fluorescent nanosensors, Nanoscale, 2017, 9(18), 5904-5911.

45 S. Tyagi, D. P. Bratu and F. R. Kramer, Multicolor molecular beacons for allele discrimination, Nat. Biotechnol., 1998, 16(1), 49-53.

46 R. E. Wang, Y. Zhang, J. Cai, W. Cai and T. Gao, Aptamerbased fluorescent biosensors, Curr. Med. Chem., 2011, 18(27), 4175-4184.

47 J. Labéguerie-Egéa, H. M. McEvoy and C. McDonagh, Synthesis, characterisation and functionalisation of luminescent silica nanoparticles, J. Nanopart. Res., 2011, 13(12), 6455-6465. 